\title{
Does the quality of residual alveolar bone apical to a periodontal lesion beneath the maxillary sinus play a vital role in preventing the extension of periodontal disease to maxillary sinus? - A CBCT assisted retrospective study
}

\author{
Vijay Apparaju ${ }^{1}$, Sunil Vaddamanu ${ }^{2}$, Bhupesh Mandali ${ }^{3}$, Rajesh Vyas ${ }^{2}$, Vishwanath \\ Gurumurthy ${ }^{2}$, and Soumya Vishwanath ${ }^{4}$ \\ ${ }^{1}$ Affiliation not available \\ ${ }^{2}$ King Khalid University \\ ${ }^{3}$ north west dental residency \\ ${ }^{4}$ All Care Dental Center
}

July 21, 2020

\begin{abstract}
Objectives: The Mucous membrane of the maxillary sinus is sensitivis susceptible to infection or inflammation adjacent to it, which may contribute to mucous membrane thickening (MMT). Residual alveolar bone quality (RABQ) is the nature of the remaining bone apical to periodontal defect adjoining to the floor of the maxillary sinus. The current study aimed to analyze the minimum RABQ to prevent the extension of periodontal pathology from reaching maxillary sinus using Cone-beam computed tomography (CBCT). Methods: In this retrospective observational study, 240 sinus exposure CBCT records of 134 patients were evaluated. Patients with at least one sinus exposure were included. RABQ and MMT were calculated using CBCT inbuilt tools. RABQ was divided into four groups based on Gray scale values (GSV). Statistical analysis was performed using One way ANOVA and independent sample t-tests. Correlation was completed applying Pearson's correlation coefficient. Results: Significant difference $(\mathrm{p}<0.05)$ was observed between the MMT values of the four RABQ groups. Inverse correlation was observed between mean MMT and GSV values. Mean MMT is higher than pathological MMT range $(>2 \mathrm{~mm})$, with significant differences in groups A and B, where mean GSV values are less than 500. Mean GSV greater than 500 in groups C and D show non-pathological MMT. Prevalence of MMT is $91.4 \%$ if GSV is $<500$ and $7.5 \%$ if GSV is $>500$. Conclusions: Our study suggests that MMT is present if RABQ has GSV values < 500. Maxillary sinusitis and its etiology from periodontal pathology can be excluded based on RABQ adjoining periodontal lesion. Early detection and prompt treatment, along with appropriate regenerative protocols can be performed to increase the RABQ. Further microbiological investigation is required to support the present results.
\end{abstract}

\section{Hosted file}

Manuscript_Full_Review.doc available at https://authorea.com/users/344712/articles/471132does-the-quality-of-residual-alveolar-bone-apical-to-a-periodontal-lesion-beneath-themaxillary-sinus-play-a-vital-role-in-preventing-the-extension-of-periodontal-disease-tomaxillary-sinus-a-cbct-assisted-retrospective-study 


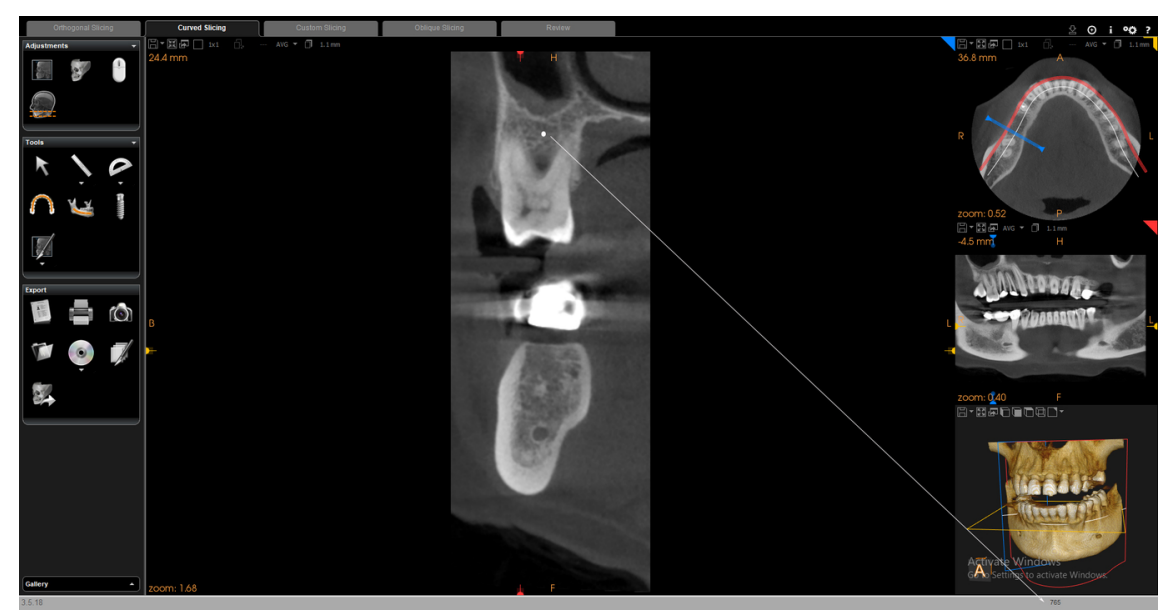




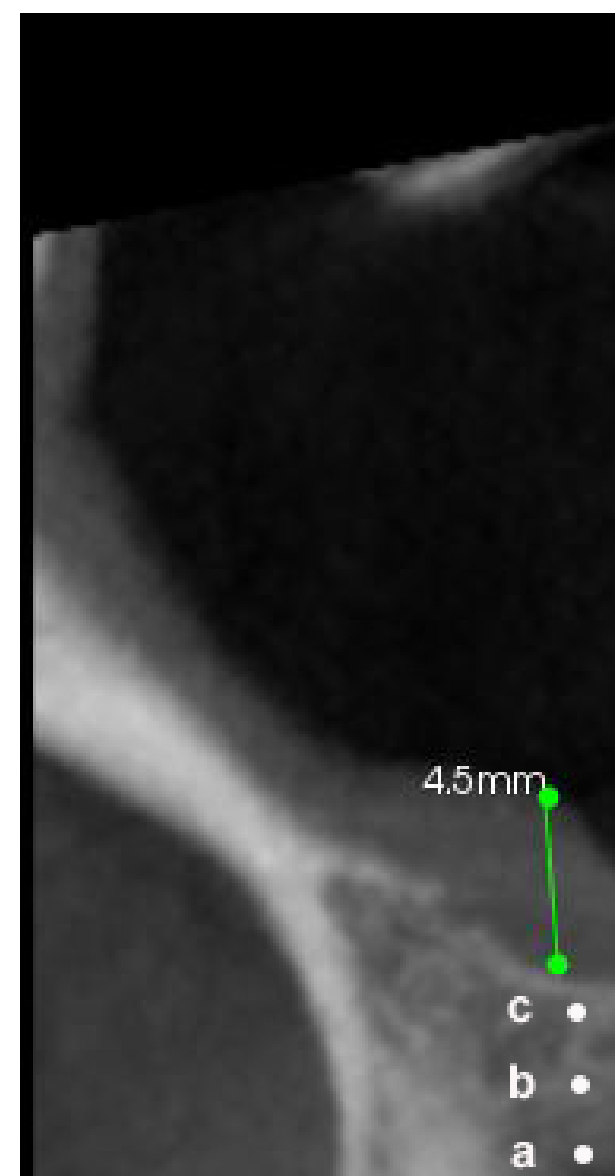




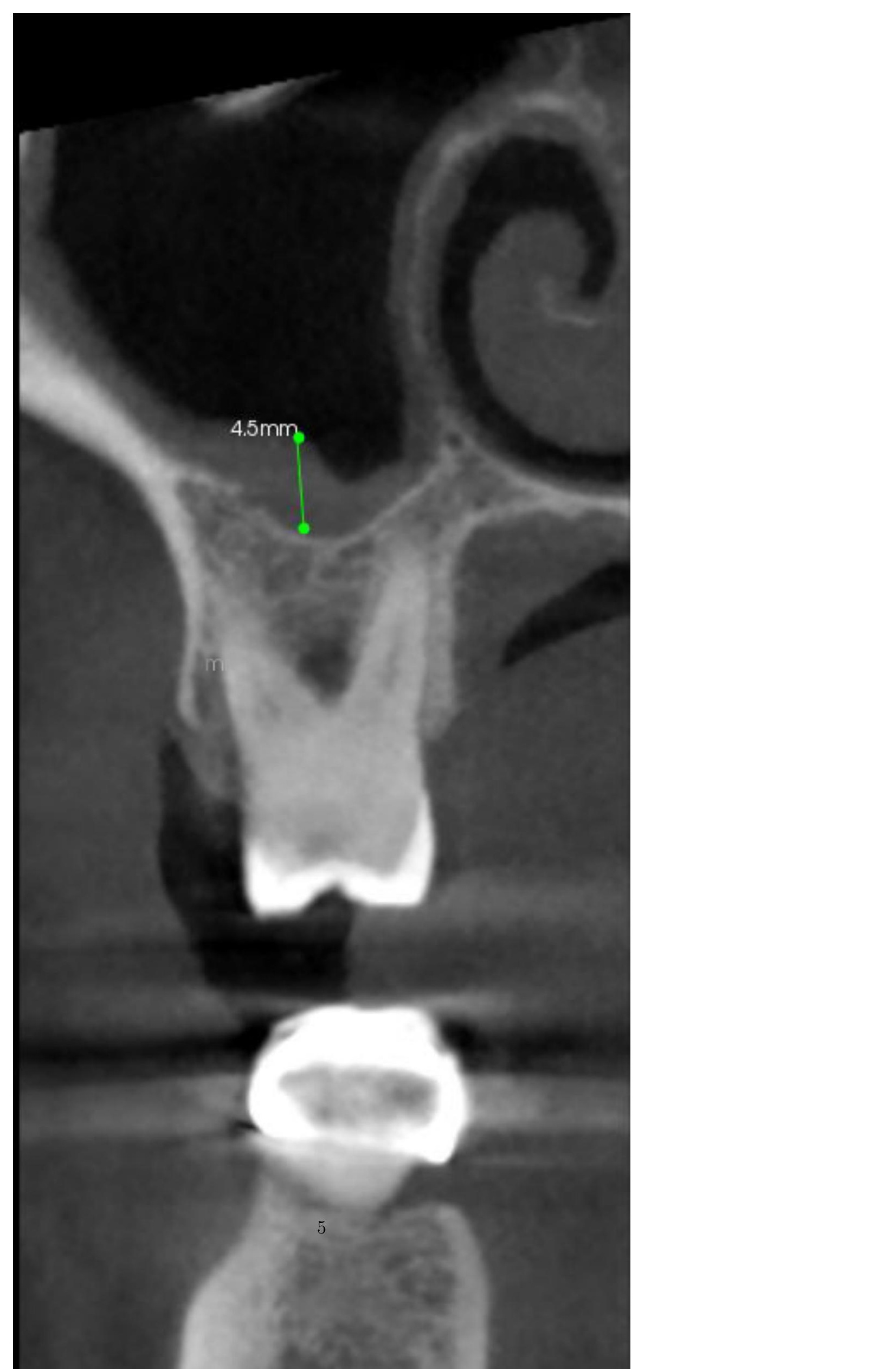




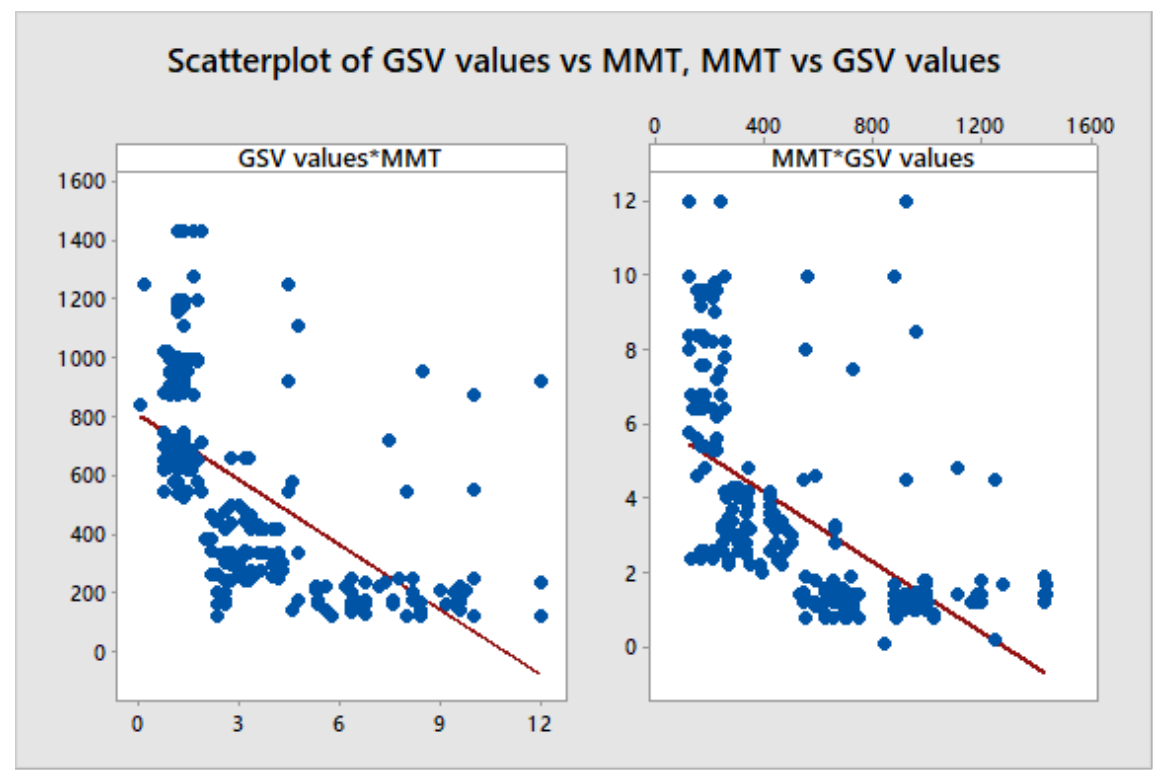

